BNL-113969-2017-JA

\title{
Flexible Multiferroic Bulk Heterojunction with Giant Magnetoelectric Coupling via the van der Waals Epitaxy
}

T. Amrillah, Y. Bitla, K. Shin, T. Yang, Y.-H. Hsieh, Y.-Y. Chiou, H.-J. Liu, T. H. Do, D. Su, Y.-C. Chen, S.-U. Jen, L.-Q. Chen, K. H. Kim, J.-Y. Juang, and Y.-H. Chu

Accepted by ACS Nano

May 2017

Center for Functional Nanomaterials

Brookhaven National Laboratory

\author{
U.S. Department of Energy \\ USDOE Office of Science (SC), \\ Basic Energy Sciences (BES) (SC-22)
}




\section{DISCLAIMER}

This report was prepared as an account of work sponsored by an agency of the United States Government. Neither the United States Government nor any agency thereof, nor any of their employees, nor any of their contractors, subcontractors, or their employees, makes any warranty, express or implied, or assumes any legal liability or responsibility for the accuracy, completeness, or any third party's use or the results of such use of any information, apparatus, product, or process disclosed, or represents that its use would not infringe privately owned rights. Reference herein to any specific commercial product, process, or service by trade name, trademark, manufacturer, or otherwise, does not necessarily constitute or imply its endorsement, recommendation, or favoring by the United States Government or any agency thereof or its contractors or subcontractors. The views and opinions of authors expressed herein do not necessarily state or reflect those of the United States Government or any agency thereof. 


\section{TABLE OF CONTENTS GRAPHIC}

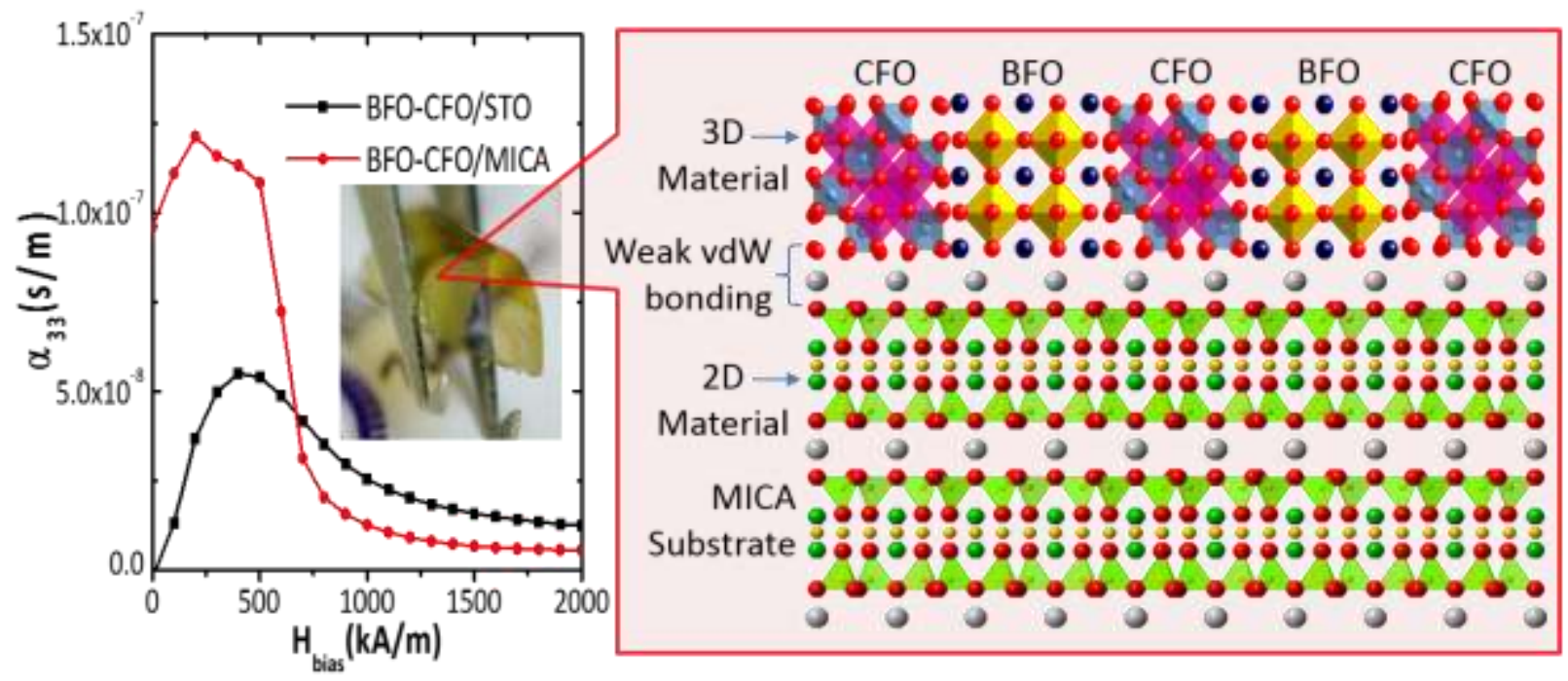

Flexible self-assembled multiferroic bulk heterojunction $\mathrm{BiFeO}_{3}-\mathrm{CoFe}_{2} \mathrm{O}_{4} / \mathrm{Muscovite}$ 


\section{Flexible Multiferroic Bulk Heterojunction with}

\section{Giant Magnetoelectric Coupling via the van der}

\section{Waals Epitaxy}

Tahta Amrillah, ${ }^{\dagger}$ Yugandhar Bitla, ${ }^{*}$ Kwangwoo Shin, ${ }^{\S}$ Tiannan Yang, ${ }^{\perp}$ Ying-Hui Hsieh, ${ }^{*}$ Yu-You Chiou, "Heng-Jui Liu, ${ }^{\Delta}$ Thi Hien Do, ${ }^{\#}$ Dong Su, ${ }^{\square}$ Yi-Chun Chen, ${ }^{\prime}$ Shien-Uang Jen, ${ }^{*}$ Long-Qing Chen, ${ }^{\perp}$ Kee Hoon Kim, ${ }^{\S}$ Jenh-Yih Juang, ${ }^{\dagger, *}$ and Ying-Hao Chu ${ }^{\dagger, \ddagger, \#, \Delta, *}$

${ }^{\dagger}$ Department of Electrophysics, National Chiao Tung University, Hsinchu 30010, Taiwan, Department of Materials Science and Engineering, National Chiao Tung University, Hsinchu 30010, Taiwan, ${ }^{\S}$ CeNSCMR, Department of Physics and Astronomy, Seoul National University, Seoul 151-747, Republic of Korea, ${ }^{\perp}$ Department of Materials Science and Engineering, Pennsylvania State University, University Park, PA 16802, United States of America,

$\|$ Department of Physics, National Cheng Kung University, Tainan 70101, Taiwan, ${ }^{\Delta}$ Department of Materials Science and Engineering, National Chung Hsing University, Taichung 40227, Taiwan, ${ }^{\#}$ Insitute of Physics, Academia Sinica, Taipei 11529, Taiwan, ${ }^{\square}$ Center for Functional Nanomaterials, Brookhaven National Laboratory, Upton, NY 11973, United States of America, ${ }^{\bullet}$ Material and Chemical Research Laboratories, Industrial Technology Research Institute, Hsinchu 31040, Taiwan. 
ABSTRACT Magnetoelectric nanocomposites have been a topic of intense research due to their profound potential in the applications of electronic devices based on the spintronic technology. Nevertheless, in spite of significant progress made in the growth of high-quality nanocomposite thin films, the substrate clamping effect still remains as a major hurdle in realizing the ultimate magnetoelectric coupling. To overcome this obstacle, an alternative strategy of fabricating selfassembled ferroelectric-ferrimagnetic bulk heterojunction on flexible muscovite via the van der Waals epitaxy is adopted. In this study, we investigated the magnetoelectric coupling in selfassembled $\mathrm{BiFeO}_{3}(\mathrm{BFO})-\mathrm{CoFe}_{2} \mathrm{O}_{4}(\mathrm{CFO})$ bulk heterojunction epitaxially grown on flexible muscovite substrate. The obtained heterojunction is composed of vertically aligned multiferroic BFO nanopillars embedding in the ferrimagnetic CFO matrix. Moreover, due to the weak interaction between the flexible substrate and bulk heterojunction, the interface is incoherent and, hence, the substrate clamping effect is greatly reduced. The phase-field simulation model also complements our results. The magnetic and electrical characterizations highlight the improvement in magnetoelectric coupling of BFO-CFO bulk heterojunction. A magnetoelectric coupling coefficient of $74 \mathrm{mV} / \mathrm{cm} \cdot$ Oe of this bulk heterojunction is larger than the magnetoelectric coefficient reported earlier on flexible substrates. Therefore, this study delivers a viable route of fabricating remarkable ME heterojunction and yet flexible electronic devices that are robust against extreme conditions with optimized performance.

KEYWORDS: Magnetoelectric · bulk heterojunction · clamping effect · van der Waals epitaxy $\cdot$ flexible 
In magnetoelectric (ME) materials, the inherent $\mathrm{ME}$ coupling allows the tuning of electric polarization (magnetization) by an external magnetic (electric) field. ${ }^{1,2}$ Since the dawn of millennium, biphasic ME composites combining superior electrical and/or magnetic properties of the constituents into multifunctional thin-film configuration through the engineered bulk heterojunction have received much attention due to their strong magnetoelectric coupling ${ }^{1-5}$ over single-phase multiferroics at room temperature. The sizeable ME effects observed in various forms of ferroelectric-ferromagnetic bulk heterojunctions have triggered extensive researches for their profound potential applications in the field of memory and sensor devices based on the spintronic technology. ${ }^{1-6}$ The ME property, manifested in terms of the magnetostrictive and piezoelectric coefficients of the constituent components, relies on the intimate elastic coupling across the interfaces, which can be further tailored by choice, design the volume fractions of components and the composite architecture. For example, a laminar multilayer film (2-2 structure) shows better ME coupling than that of particulate-matrix nanocomposite (0-3 structure) due to larger interfacial area at the bulk heterojunctions. ${ }^{1}$ However, in the multilayer configuration, the substrate clamping effect has been identified as the primary reason in reducing the structural coupling of constituent layers and, hence, limits the maximum attainable ME coupling. ${ }^{7}$ To resolve this issue, a self-assembled pillars-matrix bulk heteroepitaxy (1-3 structures) with high volume-to-interface ratio has been explored due to relatively smaller substrate clamping effect. ${ }^{1,89}$ More advanced architectures like the quasi-particles-matrix (0-3) nanocomposites $^{2}$ and the well-ordered (0-0) BFO-CFO nanodots ${ }^{3}$ show the improved ME coupling due to substantial release of substrate clamping. ${ }^{2,310}$ However, the major obstacle encountered by all these approaches in the growth of ME composites is still the clamping effect induced by the rigid substrates, which always weakens the ME coupling. ${ }^{1-3,11,12}$ Thus, to further 
advance the development of more effective magnetoelectric composites, such an inherent deficiency should be surmounted by resorting to proper substitution for rigid substrates commonly used at present.

In the present research, we demonstrate an alternative strategy capable of overcoming this key issue by fabricating an unconstrained self-assembled BFO-CFO bulk heteroepitaxy on a twodimensional (2D) layered substrate via the van der Waals (vdW) epitaxy, which does not impose stringent lattice matching of the constituent phases and substrate and can significantly reduce defect density and the substrate clamping. ${ }^{13-19}$ This salient feature is beneficial in realizing large magnetostrictive and piezoelectric coefficients, and eventually, enhances ME coupling in the self-assembled BFO-CFO heteroepitaxy grown on muscovite mica substrate. In addition, the viability of growing various functional oxides via $\mathrm{vdW}$ epitaxy on mica ${ }^{20-24}$ has been demonstrated recently for next-generation flexible devices owing to its unique set of features such as being light-weight, transparent, flexible with atomically smooth surface, chemically inert and high-temperature stability. The investigation on magnetic and electrical properties BFO$\mathrm{CFO} /$ mica heterojunction highlights the enhancement of ME coupling. Moreover, these heterojunctions are compact, lightweight and can be easily attached to conformal geometries. Thus, we believe that the present work will provide a proficient route to realize flexible yet remarkable ME heterojunction.

\section{RESULTS AND DISCUSSION}

Free-Clamping Model. The inherent strong structural coupling between BFO and CFO phases allows the change of ferroelectric polarization by an applied magnetic field. ${ }^{1,25-27}$ However, the in-plane (IP) structural coupling ${ }^{28}$ of BFO and CFO is limited by the substrate 
clamping effect compared to the ideal strain-free bulk geometry as illustrated in the schematic of Figure 1a. In our designed heterojunction shown schematically in Figure 1b, the self-assembled BFO-CFO heteroepitaxy on 2D layered muscovite relying on the weak vdW bonding between the bulk heterojunction and substrate minimizes the clamping effect and thus, it can enable effective ME effect via structural coupling. To support our hypothesis, phase-field simulation was carried out to evaluate the strain-mediated ME coupling of BFO-CFO heteroepitaxy with and without substrate clamping effect. The modeling of the strain-mediated ME coupling in thin film nanocomposites based on simple energetic arguments exhibits a significant dependence on the elastic boundary conditions on the bottom substrate and the top surface. ${ }^{29,30}$ Phase-field simulations on BFO-CFO nanocomposites grown on $\mathrm{SrTiO}_{3}$ (STO) substrate ${ }^{31}$ and membrane without substrate clamping (mica) were performed.

The phase field model considers a thin film with thickness $h=90 \mathrm{~nm}$, which consists of BFO pillars with lateral width $d=150 \mathrm{~nm}$ embedded in the CFO matrix. A periodic IP boundary condition is employed. Temporal evolution of the ferroelectric polarization $P$ in $\mathrm{BFO}$ and the magnetization $M$ in CFO is simulated by solving the time-dependent Ginzburg-Landau equation $^{31}$ and the Landau-Lifshitz-Gilbert equation using a semi-implicit Fourier-spectral method. ${ }^{32}$ The strain distribution in the nanocomposite system is obtained by solving the elastic equilibrium with eq 1

$$
\frac{\partial\left[c_{i j k l}\left(\varepsilon_{k l}-\varepsilon_{k l}^{0}\right)\right]}{\partial x_{j}}=0
$$

using a Fourier spectral iterative perturbation method ${ }^{33}$ based on Khachaturyan's theory of mesoscopic elasticity. ${ }^{34} \varepsilon^{0}$ is the stress-free strain generated by electrostriction and magnetostriction, calculated as eq 2

$$
\varepsilon_{i j}^{0}=Q_{i j k l} P_{k} P_{l}
$$


and

$$
\varepsilon_{i j}^{0}=\left\{\begin{array}{c}
\frac{3}{2} \lambda_{100}\left(m_{i} m_{j}-\frac{1}{3}\right), \quad i=j \\
\frac{3}{2} \lambda_{111} m_{i} m_{j}, \quad i \neq j
\end{array}\right.
$$

in BFO and CFO phases, respectively; $\mathbf{Q}$ and $\lambda$ are the electrostrictive coefficient of BFO and the saturation magnetostriction of CFO, respectively, taken as $Q_{11}=0.032 \mathrm{C}^{-2} \mathrm{~m}^{4}, Q_{12}=-0.016 \mathrm{C}^{-}$ ${ }^{2} \mathrm{~m}^{4}, Q_{44}=0.020 \mathrm{C}^{-2} \mathrm{~m}^{4}, \lambda_{100}=-590 \mathrm{ppm}$ and $\lambda_{111}=120 \mathrm{ppm}^{35}$

On applying an OOP magnetic field of $H=100 \mathrm{kA} / \mathrm{m}$, our simulation finds a $\sim 34 \%$ enhancement of polarization response from 0.068 to $0.091 \mathrm{C} / \mathrm{m}^{2}$ through removing the substrate clamping in a membrane nanocomposite, which is attributed to the cooperative effect of IP and OOP elastic interactions between the $\mathrm{CFO}$ and BFO phases (Figure 1c). The significant enhancement of ME coupling coefficient in the BFO-CFO composite as shown in Figure 1d highlights the importance of reducing the substrate clamping effect. Thus, one can expect that the minimal substrate clamping effect in the $\mathrm{BFO}-\mathrm{CFO} /$ mica heterojunction mediated via the weak vdW bonds can greatly enhance the ME coupling of BFO-CFO bulk heterojunction.

Structural Characterization. The samples were prepared by pulsed laser deposition (PLD). The growth conditions can be found in the methods section. The structural features were investigated by a combination of x-ray diffraction (XRD) and transmission electron microscopy (TEM). Typical XRD pattern of the sample shown in Figure 2a shows only $(l l l)$ reflections of BFO, CFO, and $\mathrm{SrRuO}_{3}(\mathrm{SRO})$ bottom electrode together with (001)-oriented mica substrate without any other orientations or secondary phases. Moreover, Figure $2 b$ shows the phi-scans of $\{200\}_{\mathrm{SRO}},\{200\}_{\mathrm{BFO}},\{400\}_{\mathrm{CFO}}$ and $\{202\}_{\text {mica }}$ diffraction peaks. The peaks of SRO, BFO, and CFO phases exhibit six-fold symmetry with respect to three-fold symmetric mica peaks 
corresponding to their cubic and hexagonal symmetries. The epitaxial relationship can be determined as $\quad(001)_{\text {mica }} / /(111)_{\mathrm{CFO}} /(111)_{\mathrm{BFO}} / /(111)_{\mathrm{SRO}} \quad$ and $[010]_{\text {mical }} / /[01 \overline{1}]_{\mathrm{CFO}} / /[01 \overline{1}]_{\mathrm{BFO}} / /[01 \overline{1}]_{\mathrm{SRO}}$. Furthermore, the heterostructure investigated by the reciprocal space mapping (RSM) in Figure 2c exhibits well-defined reciprocal diffraction peaks, showing the clear phase separation and good crystallinity of each constituent phase. By analyzing the relative $d$-spacings of all phases from their peak positions in the map, we can identify these reflections as $\mathrm{CFO}(400), \mathrm{BFO}(200)$, and $\mathrm{SRO}(200)$. Because the reciprocal map is a vector space, we can further derive that the OOP and IP vector components should be along (111) and (3-1-1) due to the resembling included angle $\left(\sim 100^{\circ}\right)$ with that in mica (202). The calculated OOP/IP $d$-spacings are $5.135 \AA / 2.472 \AA, 2.376 \AA / 1.217 \AA$, and $2.335 \AA / 1.201 \AA$ for $\mathrm{CFO}, \mathrm{BFO}$, and SRO, respectively. Compared with their bulk values $(4.846 \AA / 2.531 \AA$ for $\mathrm{CFO}$, $2.286 \AA / 1.193 \AA$ for BFO, and $2.265 \AA / 1.183 \AA$ for SRO), the estimated OOP strains of $-5.96 \%$, $-3.94 \%$ and $-1.09 \%$, while the IP strains are $-2.3 \%, 2.01 \%$, and $1.52 \%$ for CFO, BFO and SRO, respectively. The reversed sign of the IP strain states of CFO matrix and BFO pillars indicates a strong structural coupling between them. Further structural information is also made based on the results of Raman spectroscopy shown in the supplementary information Figure S1. The red shift of the vibration modes of BFO pillars on mica substrate indicates a substrate clamping release compared to the same structure on STO substrate. ${ }^{36}$

The topography was examined by atomic force microscopy (AFM) as displayed in Figure $2 \mathrm{~d}$. The inset shows that the BFO nanopillars form as triangular islands embedded in the CFO matrix similar to that grown on $\mathrm{STO}(111) .{ }^{11,12}$ In the present study, as the constituent phases grow along [111], perovskite BFO tends to form as pillars and emerges as triangular-like island in the BFOCFO bulk heterojunction. ${ }^{11}$ This is further confirmed in the microstructure examination by the 
cross-sectional TEM depicted in Figure 2e showing the BFO nanopillars embedded in the CFO matrix on mica substrate with the CFO buffer layer and SRO bottom electrode. The high resolution (HR) TEM images displayed in Figures 2f,g also show a well-defined phase separation among the component phases with sharp interfaces between them. Moreover, the FastFourier transform (FFT) patterns from the HRTEM images along [010] of the mica substrate shown in Figure $2 \mathrm{~h}$ clearly indicate that the epitaxial relationships among the constituent phases in the composite as $[100]_{\mathrm{mica}} / /[111]_{\mathrm{SRO}} / /[111]_{\mathrm{CFO}} / /[111]_{\mathrm{BFO}}$, in agreement with the XRD result. Thus, it is clear from overall Figure 2 that the BFO and CFO phases exhibit spontaneous phaseseparation as well as strong structural coupling in BFO-CFO/mica heterojunctions with welldefined epitaxy and sharp interfaces inherent to the vdW epitaxy.

Electrical and Magnetic Properties. Having demonstrated the epitaxial growth of high quality $\mathrm{BFO}-\mathrm{CFO} / \mathrm{mica}$ heterojunction, the electric and magnetic properties were further examined. From Figure 3a, the OOP converse piezoelectric coefficient, $d_{33}$, of BFO-CFO bulk heterojunction on mica $\left(76.5 \mathrm{pmV}^{-1}\right)$ is higher than that on $\mathrm{STO}(111)\left(50 \mathrm{pmV}^{-1}\right)$ grown under similar conditions. This value is also higher than the reported $d_{33}$ in (100), (110), and (111)oriented BFO-CFO/STO heterostructures $\left(50 \mathrm{pmV}^{-1}, 40 \mathrm{pmV}^{-1}\right.$ and $30 \mathrm{pmV}^{-1}$ respectively), ${ }^{12}$ suggesting the enhanced piezoelectric response of BFO pillars has been brought about by the release of the substrate constraint. The topographic and corresponding piezoresponse force microscopy (PFM) images in Figures 3b,c confirm that the electrical properties of BFO$\mathrm{CFO} /$ mica are originated from the piezoelectric response of the BFO pillars, where the OOP component of the ferroelectric polarization in the BFO pillars can be switched between two stable states (bright and dark contrast inside and outside of the square area). On the other hand, 
the magnetic properties in this system is dominated by ferrimagnetic CFO matrix as observed in (magnetic force microscopy) MFM measurement. The topographic and corresponding MFM images in Figures 3d,e shows a variation of contrast as the scanning area changes, indicating that the magnetization of the CFO matrix in the as-grown state is randomly distributed.

Furthermore, the magnetic hysteresis loops in Figure $3 \mathrm{f}$ exhibit isotropic magnetic behavior along the IP and OOP directions. This is attributed to the growth direction of CFO along [111], since the easy axis of CFO typically points to [001]. ${ }^{9,25}$ The saturated magnetization $\left(M_{\mathrm{s}}\right)$ of $\mathrm{CFO} / \mathrm{mica}$ is $\sim 237 \mathrm{emu} / \mathrm{cm}^{3}$ with the coercivity field $\left(H_{\mathrm{c}}\right)$ of $\sim 2 \mathrm{kOe}$, smaller than that obtained from epitaxial $\mathrm{CFO} / \mathrm{STO}(\sim 3 \mathrm{kOe}),{ }^{37}$ possibly due to the effective relaxation of clamping from the mica substrate. ${ }^{3}$ Moreover, Figure $3 \mathrm{~g}$ shows the magnetostriction of the sample probed through the deflection $(\Delta z)$ of the sample under magnetic field by the conventional magnetostriction measurement using the digital holographic microscope (DHM) method. ${ }^{38}$ The magnetostriction deflection profile $\left(\Delta z_{\|}\right.$or $\left.\Delta z_{\perp}\right)$ mapped out from the fixed end of the film plane with the magnetic fields applied parallel $\left(H_{\|}\right.$, the longitudinal field $)$and perpendicular $\left(H_{\perp}\right.$, the transverse field) to the IP-direction of the sample are represented in the inset of Figure 3g. Upon the application of magnetic field $(\mathrm{H}=2000 \mathrm{Oe})$, the sample deflects up and down from the reference position $(H=0)^{38}$ as shown in Figure $3 \mathrm{~g}$. From the deflection profile, the longitudinal and transverse magnetostriction coefficients of the sample can be determined by using eq $3 .{ }^{39}$

$$
\lambda_{\|, \perp}=\left(\frac{1}{3}\right)\left[\frac{\Delta Z_{\|, \perp}}{L^{2}}\right] K \quad, K=\left(\frac{E_{s}}{E_{f}}\right)\left(\frac{t_{s}^{2}}{t_{f}}\right)\left[\frac{\left(1+v_{f}\right)}{\left(1+v_{s}\right)}\right]
$$

where $L$ is the length, $E_{\mathrm{f}}$ is the Young's modulus of the film, $E_{\mathrm{S}}$ is the Young's modulus of the substrate, $t_{\mathrm{f}}$ is film thickness, $t_{\mathrm{s}}$ is substrate thickness, $v_{\mathrm{f}}$ is the Poisson's ratio of the film, and $v_{\mathrm{s}}$ is the Poisson's ratio of the substrate, respectively. The calculated longitudinal and transverse magnetostriction coefficients are $-51 \mathrm{ppm}$ and $61 \mathrm{ppm}$, respectively (with $\Delta \lambda=\lambda_{\|}-\lambda_{\perp}=-112$ 
ppm), slightly higher than that of CFO/mica $(\Delta \lambda=-104 \mathrm{ppm}),{ }^{20}$ which can be attributed to the strong antiferromagnetic-ferrimagnetic coupling of BFO-CFO. ${ }^{8,40}$ This result is also in agreement with the previous reports that $\mathrm{CFO}$ has negative longitudinal magnetostriction and positive transverse magnetostriction. ${ }^{1,25}$

Magnetoelectric Properties. After realizing the manifestation of reduced substrate constraint in the improved electrical and magnetic properties of $\mathrm{BFO}-\mathrm{CFO} / \mathrm{mica}$, it is important to ascertain whether the same gets reflected in the magnetoelectric response. To probe the magnetoelectric properties in nanoscale, the effect of magnetic field on the electrical properties was investigated by measuring hysteresis loops $\left(d_{33}\right.$ vs $\left.V\right)$ from individual switching of BFO pillars with $(H=$ $2000 \mathrm{Oe})$ and without the applied magnetic field along the OOP-direction. The $d_{33}$ decreases with the applied magnetic field from $76.5 \mathrm{pmV}^{-1}$ to $42.5 \mathrm{pmV}^{-1}$ as shown in Figure 4a. Such a behavior is attributed to that the CFO matrix together with BFO pillars are compressed along the OOP-direction via their strong structural coupling when the magnetic field is applied along the OOP-direction due to a negative longitudinal magnetostriction of CFO matrix. As a consequence, the $d_{33}$ should decrease due to the rotation of $P$ with decreasing length of the BFO pillars along the OOP-direction. ${ }^{1-3,25-27}$ It is also shown in the PFM map with spectroscopy in the supplementary information of Figure S2 where the piezo-response signal of BFO pillars get suppressed in the applied magnetic field of 2000 Oe along the OOP-direction. The associated magnetoelectric coefficient $\left(\alpha_{\mathrm{ME}}\right)$ can be calculated by $\alpha_{\mathrm{ME}}=\Delta E / \Delta H$ with the variation in the electric field $\Delta E$ induced by the magnetic field $\Delta H\left(2000\right.$ Oe), where $\Delta E=\Delta u /\left(d_{33} \cdot D\right), \Delta u$ is the piezo-response displacement with and without magnetic bias, $D$ is the thickness of BFO-CFO nanocomposite $(\sim 200 \mathrm{~nm})$, and the corresponding $d_{33}$ without magnetic bias $(76.5 \mathrm{pm} / \mathrm{V})$. $^{2,3,10}$ 
The calculated $\alpha_{\mathrm{ME}}$ of BFO-CFO/mica is $\sim 11000 \mathrm{mV} / \mathrm{cm} \cdot \mathrm{Oe}$, which is much higher than that found in the quasi (0-3) BFO-CFO nanocomposite on rigid STO substrate $(338 \mathrm{mV} / \mathrm{cm} \cdot \mathrm{Oe}),{ }^{2}$ and comparable with the result from the unclamped PZT/CFO core-shell nanofiber structures (29500 $\mathrm{mV} / \mathrm{cm} \cdot \mathrm{Oe}) .{ }^{10}$ This observation underscores the importance of substrate clamping release in the enhancement of the ME coupling of BFO-CFO nanocomposite.

However, this converse $\alpha_{\mathrm{ME}}$ estimate based on microscopic measurements by PFM cannot reveal the direct ME response in the device level. To obtain better insights about the ME effect at the macroscopic level, the quantitative longitudinal and transverse magnetoelectric susceptibility (MES) studies that directly probe the ME effect were performed as shown in Figure S3a. Direct measurements of the MES based on a conventional scheme that employs small $H_{\mathrm{ac}}$ values with variations in the dc magnetic field $\left(H_{\mathrm{dc}}\right)$ would be useful for understanding the magnetoelectric coupling of numerous magnetoelectric films or nanostructures at the quantitative level. ${ }^{1}$ The temperature evolution of longitudinal and transverse MES are presented in Figure 4b. The longitudinal MES $\left(\alpha_{33}\right)$ is slightly smaller than transverse $\operatorname{MES}\left(\alpha_{31}\right)$ at all temperatures due to the fact that spontaneous polarization of BFO pillars point along the OOP direction while CFO matrix exhibits magnetic isotropy, and thus the negative sign is attributed from overall negative magnetostriction of $\mathrm{CFO}^{1}$ consistent with the previous microscopic magnetoelectric measurement result. This is in stark contrast with anisotropic MES in BFO-CFO/STO where magnetic anisotropy of CFO plays a key role. ${ }^{1}$ Notably, the room temperature MES of BFO$\mathrm{CFO} / \mathrm{mica}$ is about $\sim 160 \mathrm{ps} / \mathrm{m}(\sim 74 \mathrm{mV} / \mathrm{cm} \cdot$ Oe) higher than BFO-CFO/STO (001) $(\sim 130 \mathrm{ps} / \mathrm{m}$ $(\sim 60 \mathrm{mV} / \mathrm{cm} \cdot \mathrm{Oe}))^{1}$ displaying similar (1-3) nanostructure. The enhancement (24\%) in MES of $\mathrm{BFO}-\mathrm{CFO} /$ mica is in good agreement with the phase-field simulation prediction presented in Figure 1 confirming the reduced mica substrate clamping effect brought about by the vdW 
epitaxy. Moreover, Table 1 compares the magnetoelectric coefficients from various magnetoelectric composites for various material combinations and different architectures aimed at achieving best magnetoelectric response. The enhancement in reported magnetoelectric coupling is mainly related to the substrate clamping release which strongly depends on the architecture and also the suitable composite components. The BFO-CFO/mica system shows a better magnetoelectric coefficient mainly due to the release of the mica substrate clamping to achieve an effective strain-coupling.

Flexibility. Finally, it is worthwhile to mention that, the magnetoelectric coefficient of BFOCFO on mica is larger than the magnetoelectric coefficient reported on flexible substrate due to superior crystalline quality and nanoscale control of the interface connectivity. ${ }^{1,2,3}$ The effect of mechanical strain on the physical properties of the current flexible ME nanocomposite is tested further. The M-H loop remained unaltered under the different bending modes (bending radius $\sim 2$ $\mathrm{mm}$ ) as shown in Figure 5a in agreement with the earlier work $\mathrm{CFO} / \mathrm{mica} .{ }^{20}$ In contrast, the $d_{33}$ of each selected individuals BFO pillars gets affected when the sample is under tensile and compressive bend as shown in Figure 5b, which is attributed to the rotation of $P$ due to the variation of length of BFO pillars along $c$-axis under bending. The magnetic field of 0.1 and 0.2 T reduces the $d_{33}$ coefficients further. The corresponding $\alpha_{\mathrm{ME}}$ estimated from $d_{33}(H=0,0.1,0.2$ T) as a function of bending are depicted in Figure 5c. It is noteworthy that the obtained microscopic $\alpha_{\mathrm{ME}}$ values under bending are still comparable to those observed in unclamped magnetoelectric composites ${ }^{3,10}$ and higher than quasi (0-3) structure BFO-CFO/STO ${ }^{2}$ calculated with similar method. The variation of local electric response of the BFO pillars under mechanical strain is responsible for the variation of $\alpha_{\mathrm{ME}}$. However, the $\alpha_{\mathrm{ME}}$ varies much slower than local $d_{33}$ 
under mechanical strain. The transversal MES $\left(\alpha_{31}\right)$ variation as a function of bending radius is further tested, the $\alpha_{31}$ remains constant even under a small bending radius of $5 \mathrm{~mm}$ as shown in Figure S4. A permanent damage of the sample at the bending radius of $4 \mathrm{~mm}$ can be detected when the magnetoelectric coupling behavior vanishes. Furthermore, the microscopic magnetoelectric measurements for various bending cycles under a bending radius of $\sim 7 \mathrm{~mm}$ have been carried out to check the recoverability of ME coefficient as displayed in Figure S5. The result shows a small variation of the $\alpha_{\mathrm{ME}}$ up to 1000 bending cycles indicating that the $\mathrm{ME}$ coupling of this system is robust against mechanical bending. The insensitivity of magnetoelectric response under mechanically bending is attributed to the stability of magnetostricition of the CFO matrix (Figure 5a). The robust magnetoelectric behavior against mechanical strain makes this heterojunction a great candidate for flexible electronic devices applications.

\section{CONCLUSIONS}

In summary, we have successfully fabricated a high-quality BFO-CFO bulk heterojunction on mica substrate with minimal substrate clamping. Consistent with the phase-field simulations of the free clamping model, this nanocomposite exhibits the enhanced magnetic and electric properties. The most striking feature of such a flexible bulk heterojunction is the ME performance better than the conventionally reported of flexible ME nanocomposites. Our approach provides a great direction in the realization of highly sensitive yet flexible ME electronic devices application with optimized performance.

\section{METHODS}


Materials. The self-assembled BFO-CFO heterojunction with the thickness of $200 \mathrm{~nm}$ was grown on mica substrate via PLD technique using a mixed BFO-CFO target with the volume fraction ratio of $65: 35$. The mica substrate $\left(\mathrm{KAl}_{2}\left(\mathrm{AlSi}_{3} \mathrm{O}_{10}\right)(\mathrm{OH})_{2}\right)$, monoclinic structure with $a=$ $5.253 \AA, b=9.086 \AA, c=10.159 \AA, \beta=99.9^{\circ}$ (space group $\mathrm{C} 12 / c 1$ ) was heated up to $650{ }^{\circ} \mathrm{C}$ and kept in an oxygen pressure of 100 mTorr during the growth. Prior to the deposition of BFO-CFO heteroepitaxy on mica, a CFO interfacial buffer layer was deposited. Then, the SRO bottom electrode was grown on top of CFO interfacial buffer layer for electrical and magnetoelectric measurements. In the meantime, a reference sample on STO(111) substrate was also prepared in the same growth conditions.

Measurement. XRD with phi scan, RSM, AFM, and TEM were employed to study the details of the structure and morphology of the samples while the Raman spectroscopy is used to reveal the release of substrate clamping effect. The magnetic and magnetostrictive properties were investigated by a Quantum Design ${ }^{\circledR}$ magnetic property measurement system (MPMS), a superconducting quantum-interference device (SQUID) magnetometer, MFM, and DHM method. Furthermore, PFM has been used to characterize the piezoelectric response of samples by using a Ptlr-coated tip with an AC voltage of $1.0 \mathrm{~V}$ modulated at $6.39 \mathrm{kHz}$. The macroscopic and microscopic ME responses were measured using a highly sensitive home-built magnetoelectric susceptometer operating inside the PPMS (Quantum Design) and PFM with applied magnetic field, respectively. 
FIGURES

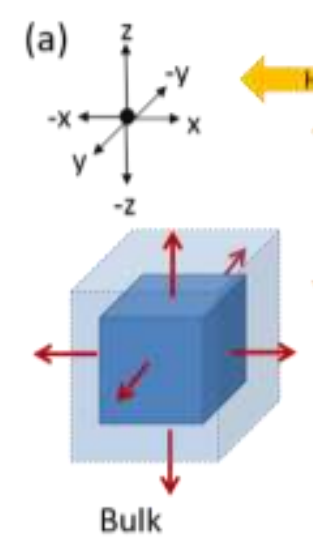

(c)
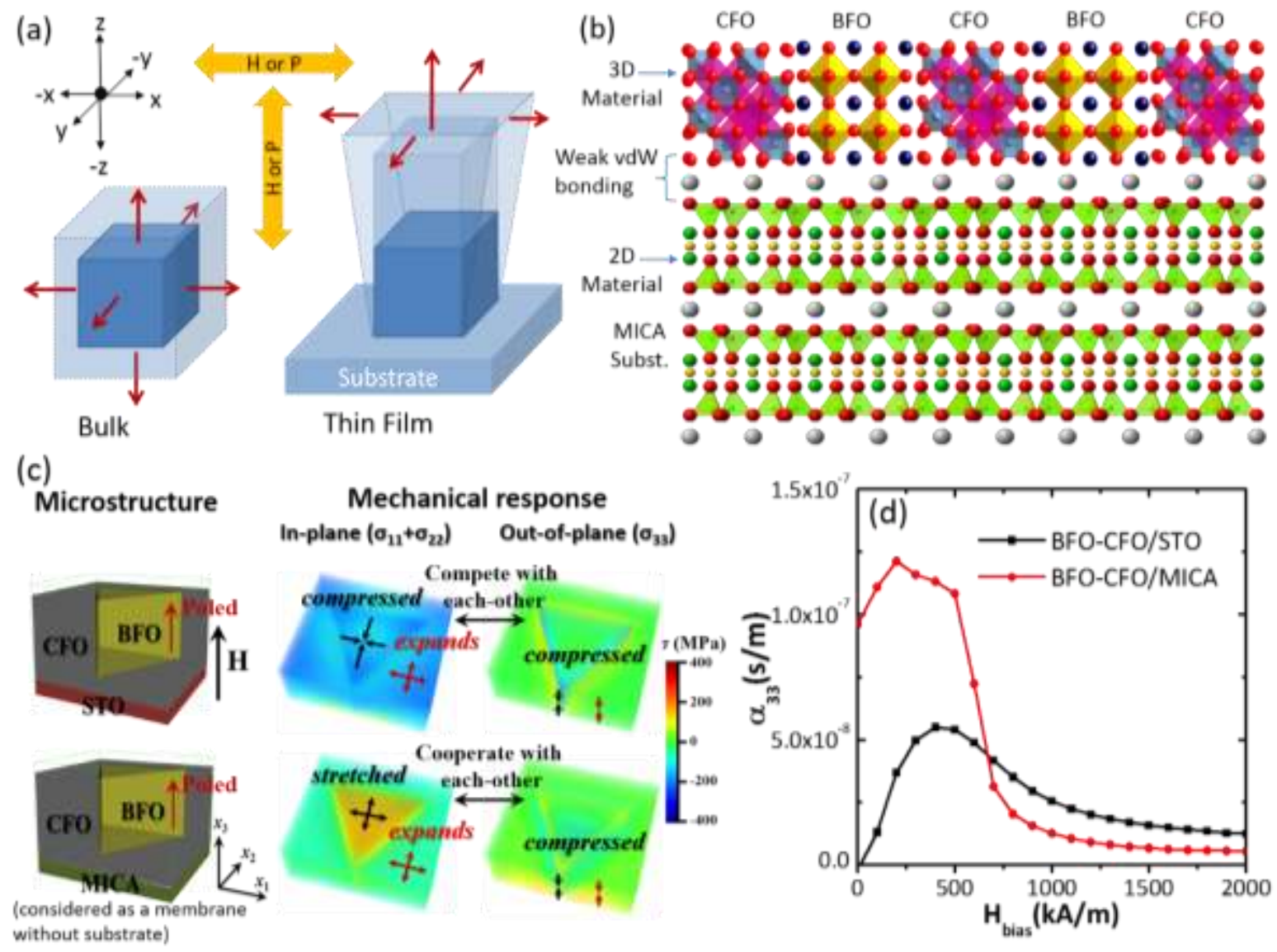

Figure 1. (a) Schematic of elasticity direction of the bulk and film (adopted from ref. [28]), shows that the elasticity of the film is limited due to clamping effect from the rigid substrate. (b) Crystal structure of the interface region between the CFO-BFO composite and MICA substrate, with 2D-3D bonding which exhibits the vdW heteroepitaxial relationship. (c) Phase structure of the BFO-CFO films in the phase-field model and simulated stress distribution on applying an out-of-plane (OOP) magnetic field of $H=100 \mathrm{kA} / \mathrm{m}$. (d) Longitudinal magnetoelectric coupling 
coefficient $\alpha_{33}$ simulated using a phase-field model, which shows an enhancement of over $100 \%$ at low magnetic field bias $(<500 \mathrm{kA} / \mathrm{m})$ on removing the substrate clamping.
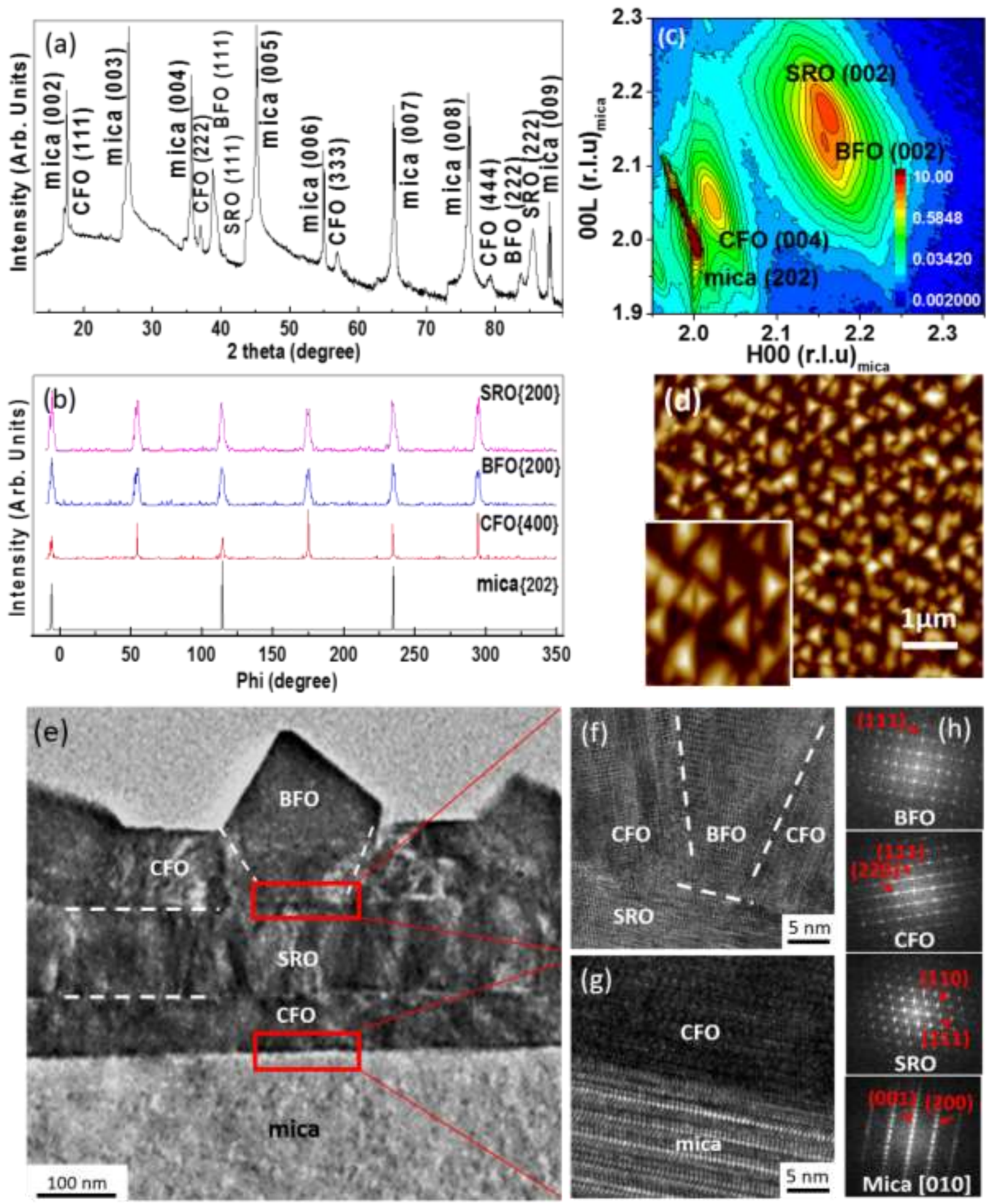
Figure 2. (a) XRD of BFO-CFO/SRO/CFO/mica. (b) Phi-scans of $\mathrm{SRO}\{200\}, \mathrm{BFO}\{200\}$, CFO $\{400\}$ and mica $\{202\}$. (c) The RSM result shows incoherent epitaxy of BFO and CFO, evidencing no significant clamping effect from mica substrate. (d) The surface morphology of $\mathrm{BFO}-\mathrm{CFO} / \mathrm{SRO} / \mathrm{CFO} /$ mica by $\mathrm{AFM}$, indicates the $\mathrm{BFO}$ triangular pillars embedded in the $\mathrm{CFO}$ matrix. (e) The cross-sectional TEM image shows the BFO-CFO film, SRO and CFO layer and also the mica substrate. (f) and (g) are the HRTEM images of the BFO-CFO/SRO and CFO/mica interfaces, respectively. (h) The FFT patterns patterns indicate the orientation relation between constituent phases and the substrate.
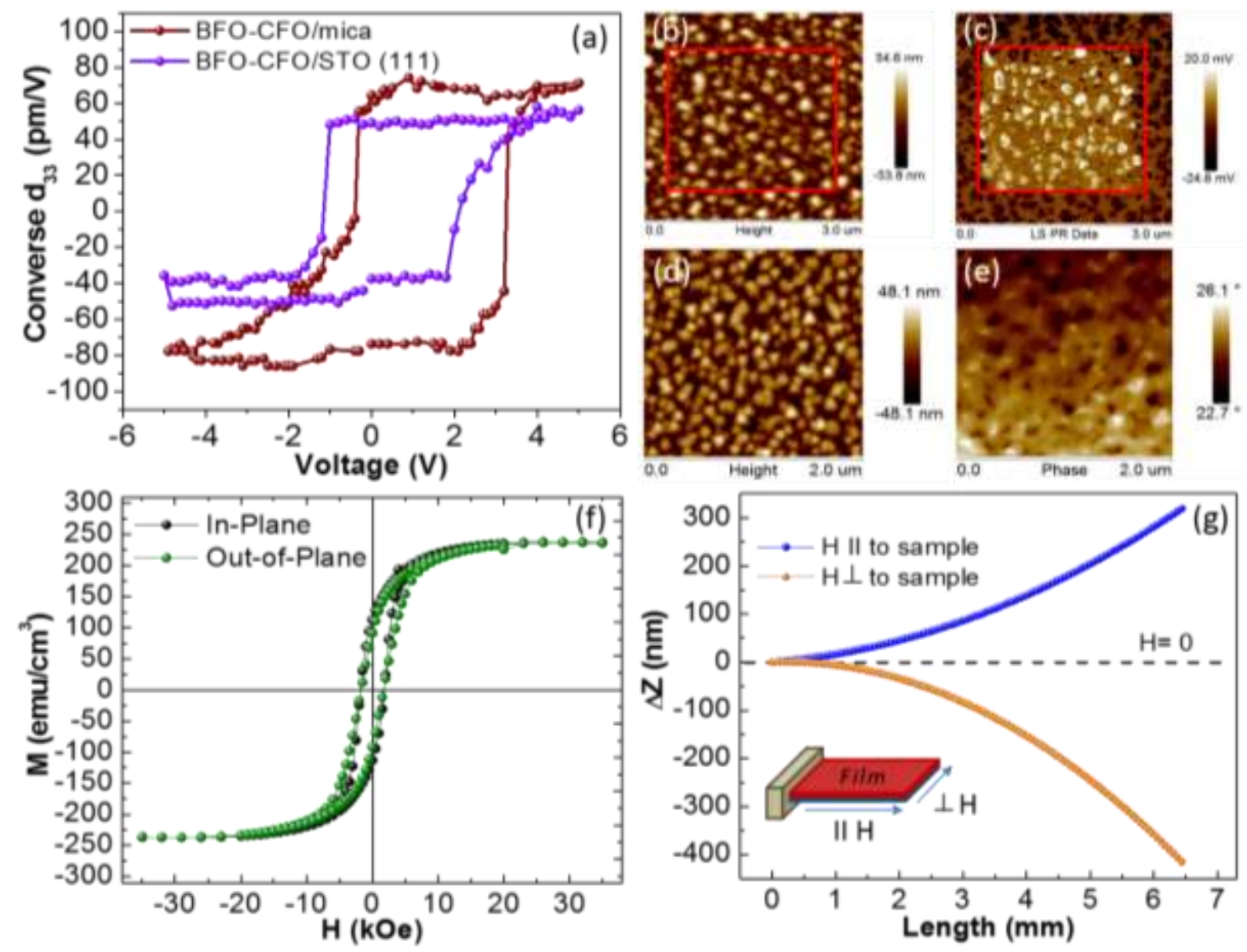
Figure 3. (a) Comparison of piezo-response from BFO-CFO grown on mica (brown) and STO (111) (purple) substrate. (b) and (c) show the topography and electric properties probed by PFM exhibit the ferro/piezoelectric response of BFO pillars. (d) and (e) show that the topography and MFM to illustrate that the magnetic properties are dominated by $\mathrm{CFO}$ matrix. (f) The M-H hysteresis loops show that the composite is magnetically isotropic. (g) The magnetostriction measurement to observe the magnetostriction of the sample in respect with the deflection behavior.
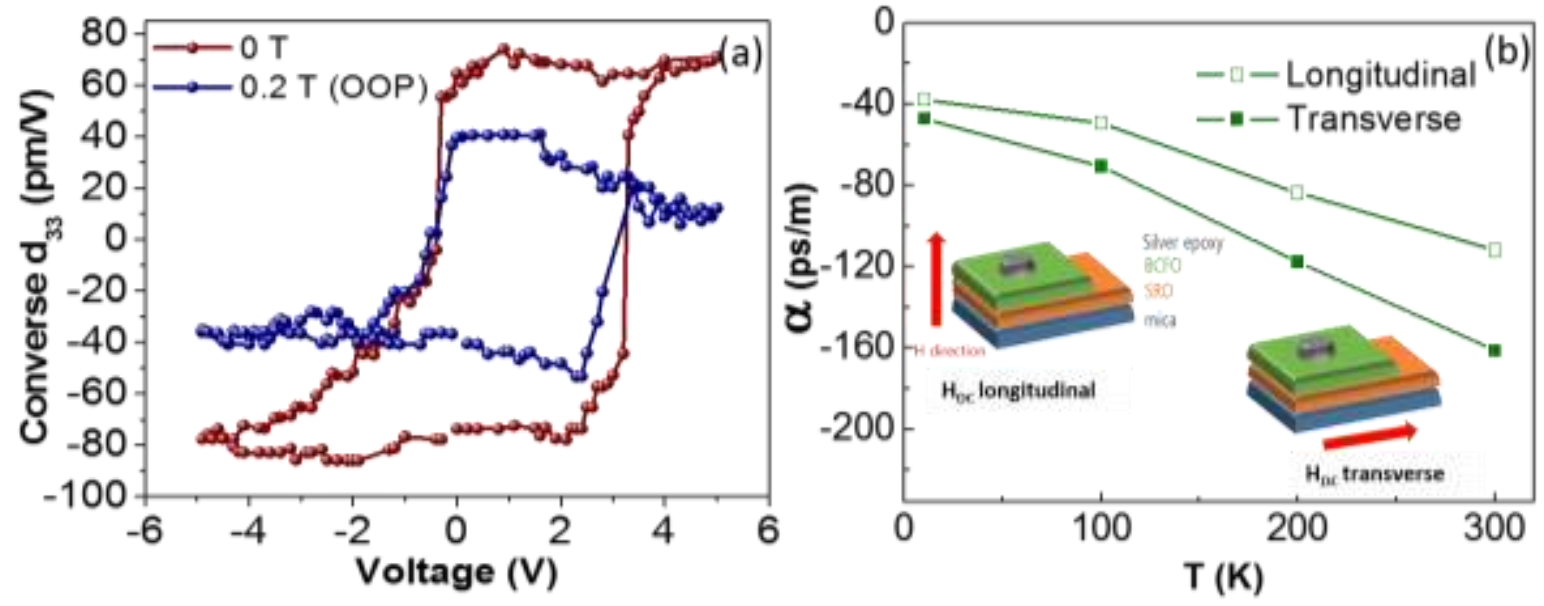

Figure 4. (a) $d_{33}$ vs. V from the switching of BFO pillars with and without applied magnetic field along the OOP direction. (b) The MES variation at $10 \mathrm{~K}, 100 \mathrm{~K}, 200 \mathrm{~K}$, and $300 \mathrm{~K}$. 

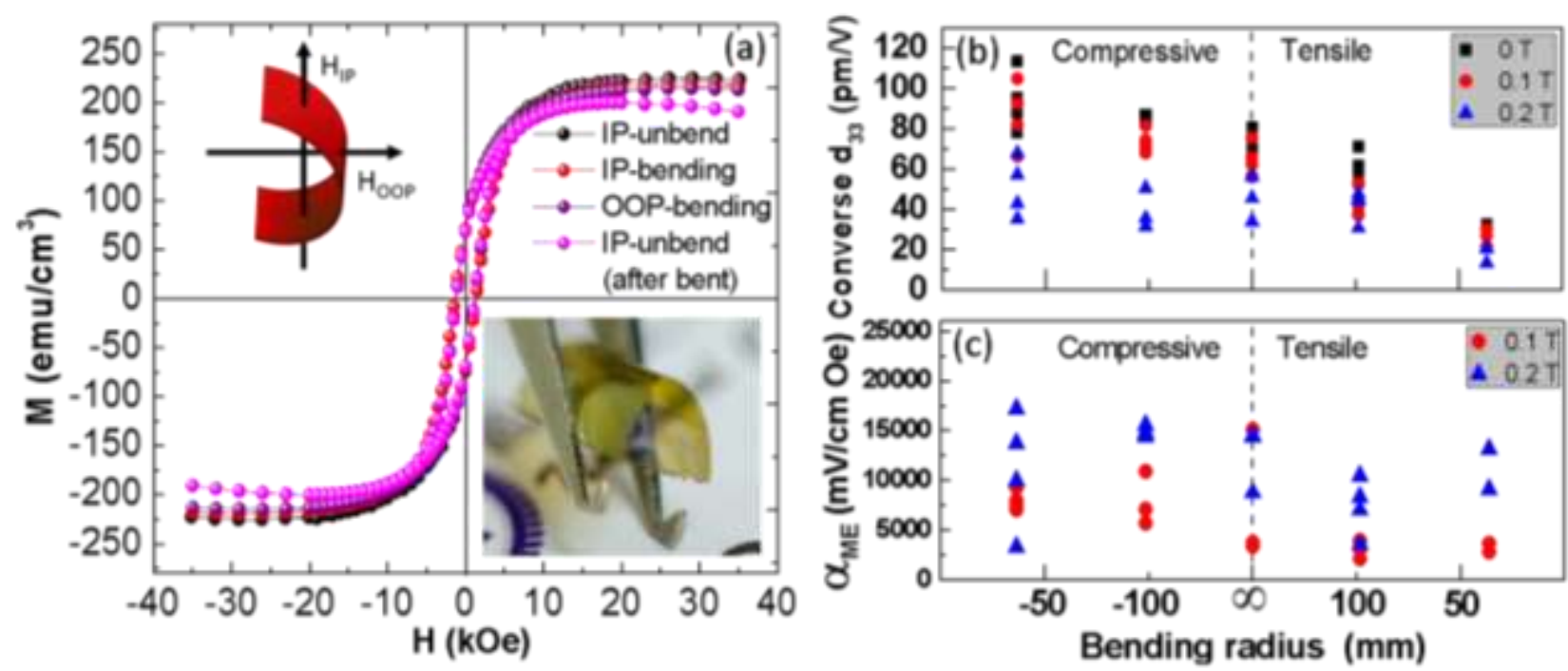

Figure 5. (a) The M-H loop of BFO-CFO with under bending modes. (b) The $d_{33}$ of each selected individuals BFO pillars under different bending radius with and without applied magnetic field. (c) Calculated $\alpha_{\mathrm{ME}}$ from Figure $5 \mathrm{~b}$.

TABLE

Table 1. Comparison of magnetoelectric coefficient from magnetoelectric composite materials

\begin{tabular}{|c|c|c|c|c|c|}
\hline \multicolumn{5}{|c|}{ Microscopic $\alpha_{\mathrm{ME}}$ measurements by PFM } \\
\hline Architecture & Subst. & \multicolumn{2}{|c|}{ Component phases } & $\alpha_{\mathrm{ME}}(\mathrm{mV} / \mathrm{cm} \cdot$ Oe) & Ref. \\
\hline Quasi (0-3) & STO & BFO matrix & CFO particles & 338 & {$[2]$} \\
structure & & & & & \\
\hline Well-ordered 0-0 & STO & BFO nanodot & CFO nanodot & 12000 & {$[3]$} \\
nanodot & & & & & \\
\hline
\end{tabular}




\begin{tabular}{|c|c|c|c|c|c|}
\hline $\begin{array}{c}\text { Core- } \\
\text { ShellNanofibers }\end{array}$ & - & PZT & $\mathrm{CFO}$ & 29500 & [10] \\
\hline $1-3$ structure & Mica & CFO matrix & BFO pillars & 11000 & $\begin{array}{l}\text { This } \\
\text { work }\end{array}$ \\
\hline \multicolumn{6}{|c|}{ Macroscopic $\alpha_{\mathrm{ME}}$ measurements by magnetoelectric susceptometer } \\
\hline Architecture & Subst. & \multicolumn{2}{|c|}{ Component phases } & $\alpha_{\mathrm{ME}}(\mathrm{mV} / \mathrm{cm} \cdot \mathrm{Oe})$ & Ref. \\
\hline $2-2$ structure & LAO & PZT layer & LSMO layer & 4.2 & [41] \\
\hline $2-2$ structure & STO & BTO layer & CFO layer & 66 & [42] \\
\hline $2-2$ structure & STO & PZT layer & NFO layer & 20 & [43] \\
\hline $0-3$ structure & STO & PZT matrix & NFO particles & 16 & [44] \\
\hline $1-3$ structure & STO & BFO matrix & CFO pillars & 60 & [1] \\
\hline 2-2 structure & $\mathrm{Ni}$ foil & $\mathrm{BFO}$ & $\mathrm{Ni}$ & 3.5 & [45] \\
\hline 2-2 structure & $\begin{array}{c}\text { Metglass } \\
\text { foil }\end{array}$ & BTO & Metglass & 55 & [46] \\
\hline 2-2 structure & $\begin{array}{c}\text { flexible } \\
\text { polyimide }\end{array}$ & $\mathrm{CFO}$ & $\mathrm{P}(\mathrm{VDF}-\mathrm{TrFE})$ & 12 & [47] \\
\hline $1-3$ structure & Mica & CFO matrix & BFO pillars & 74 & $\begin{array}{l}\text { This } \\
\text { work }\end{array}$ \\
\hline
\end{tabular}




\section{ASSOCIATED CONTENT}

Supporting Information Available: Additional Figures and data as described in the text. This material is available free of charge via the Internet at http://pubs.acs.org.

\section{AUTHOR INFORMATION}

\section{Corresponding Author}

*jyjuang@cc.nctu.edu.tw, yhc@nctu.edu.tw.

\section{Author Contributions}

T. A., Y. B., and Y. H.C. designed the experiments. T. A. prepared all the samples in this study, performed XRD, AFM, and SQUID measurement and designed all schematic. K. S. and K. H. K. were responsible for MES measurement and analysis. T. Y. and L. Q. C. gave the phase field model and schematic. Y. H. H., Y. Y. C., and Y. C. C., carried out the PFM, MFM, and Raman measurement and analysis. H. J. L. performed the RSM measurement and analysis. D. T. H. performed the phi scan. D. S. responsible for TEM result. S. U. J. responsible for DHM result. T. 
A., Y. B., J. Y. J., and Y. H. C. designed the study and wrote the manuscript with the help of all authors. All authors discussed the results and Y. H. C. directed the study.

\section{ACKNOWLEDGMENT}

The authors gratefully acknowledge the financial support by the Ministry of Science and Technology, R.O.C. (Grant No. MOST 103-2112-M-009-015-MY3, MOST 104-2628-E-009005-MY2, and MOST 104-2923-M-009-005-MY2). The work at Seoul National University was supported by the National Creative Research Initiative (2010-0018300) and the Korea-Taiwan Cooperation Program (0409-20150111). The work at Pennsylvania State University was supported by NSF under Grant No. DMR-1410714.

\section{REFERENCES AND NOTES}

1. Oh, Y. S.; Crane, S.; Zheng, H.; Chu, Y. H.; Ramesh, R.; Kim, K. H. Quantitative Determination of Anisotropic Magnetoelectric Coupling in $\mathrm{BiFeO}_{3}-\mathrm{CoFe}_{2} \mathrm{O}_{4}$ Nanostructures. Appl. phys. Lett. 2010, 97, 052902.

2. Li, Y.; Wang, Z.; Yao, J.; Yang, T.; Wang, Z.; Hu, J. M.; Chen, C.; Sun, R.; Tian, Z.; Li, J.; Chen, L. Q.; Viehland, D. Magnetoelectric Quasi-(0-3) Nanocomposite Heterostructures. Nat. Commun. 2015, 6, 6680.

3. Tian, G.; Zhang, F.; Yao, J.; Fan, H.; Li, P.; Li, Z.; Song, X.; Zhang, X.; Qin, M.; Zeng, M.; Zhang, Z.; Yao, J.; Gao, X.; Liu, J. Magnetoelectric Coupling in Well-Ordered Epitaxial $\mathrm{BiFeO}_{3} / \mathrm{CoFe}_{2} \mathrm{O}_{4} / \mathrm{SrRuO}_{3}$ Heterostructured Nanodot Array. ACS Nano 2016, $10,1025-1032$. 
4. Nan, C. W.; Bichurin, M. I.; Dong, S. X.; Viehland, D.; Srinivasan, G. Multiferroic Magnetoelectric Composites: Historical Perspective, Status, and Future Directions. J. Appl. Phys. 2008, 103, 031101.

5. Yu, C.; Pechan, M. J.; Srivastava, S.; Palmstrøm, C. J.; Biegaslski, M.; Brooks, C.; Schlom, D. Ferromagnetic Resonance in Ferromagnetic/Ferroelectric Fe/BaTiO $3 / \mathrm{SrTiO}_{3}$ (001). J. Appl. Phys. 2008, 103, $07 \mathrm{~B} 108$.

6. Wang, Y.; Li, J.; Viehland, D. Magnetoelectrics for Magnetic Sensor Applications: Status, Challenges and Perspectives. Mater. Today 2014, 17, 269-275.

7. Nan, C. W.; Liu, G.; Lin, Y. H.; Chen, H. D. Magnetic-Field-Induced Electric Polarization in Multiferroic Nanostructures. Phys. Rev. Lett. 2005, 94, 197203.

8. Chen, Y. J.; Hsieh, Y. H.; Liao, S. C.; Hu, Z.; Huang, M. J.; Kuo, W. C.; Chin, Y. Y.; Uen, T. M.; Juang, J. Y.; Lai, C. H.; Lin, H. J.; Chen, C. T.; Chu, Y. H. Strong Magnetic Enhancement in Self-Assembled Multiferroic-Ferrimagnetic Nanostructures. Nanoscale 2013, 5, 4449-4453.

9. Liao, S. C.; Tsai, P. Y.; Liu, H. J.; Yang, J. C.; Lin, S. J.; Lai, C. H.; Chu, Y. H. Misorientation Control and Functionality Design of Nanopillars in Self-Assembled Perovskite-Spinel Heteroepitaxial Nanostructure. ACS Nano 2011, 5, 4118-4122.

10. Xie, S. H.; Ma, F. Y.; Liu, Y. M.; Li, J. Y. Multiferroic $\mathrm{CoFe}_{2} \mathrm{O}_{4}-\mathrm{Pb}\left(\mathrm{Zr}_{0.52} \mathrm{Ti}_{0.48}\right) \mathrm{O}_{3}$ Core-Shell Nano Fibers and Their Magnetoelectric Coupling. Nanoscale 2011, 3, 3152-3158. 
11. Zheng, H.; Zhan, Q.; Zavaliche, F.; Sherburne, M.; Straub, F.; Cruz, M. P.; Chen, L. Q.; Dahmen, U.; Ramesh, R. Controlling Self-Assembled Perovskite-Spinel Nanostructures. Nano Lett. 2006, 6, 1401-1407.

12. Yan, L.; Wang, Z.; Xing, Z.; Li, J.; Viehland, D. Magnetoelectric and Multiferroic Properties of Variously Oriented Epitaxial $\mathrm{BiFeO}_{3}-\mathrm{CoFe}_{2} \mathrm{O}_{4}$ Nanostructured Thin Films. J. Appl. Phys. 2010, 107, 064106.

13. Geim, A.; Grigorieva, I. Van der Waals heterostructures. Nature 2013, 499, 419-425.

14. Koma, A.; Sunouchi, K.; Miyajima, T. Fabrication and Characterization of Heterostructures with Sub-Nanometer Thickness. Microelectron. Eng. 1984, 2, 129 136.

15. Utama, M. I. B.; Zhang, Q.; Zhang, J.; Yuan, Y.; Belarre, F. J.; Arbiol, J.; Xiong, Q. Recent Developments and Future Directions in the Growth of Nanostructures by Van Der Waals Epitaxy. Nanoscale 2013, 5, 3570-3588.

16. Kim, J.; Bayram, C.; Park, H.; Cheng, C. W.; Dimitrakopoulos, C.; Ott, J. A.; Reuter, K. B.; Bedell, S. W.; Sadana, D. K. Principle of Direct Van Der Waals Epitaxy of Single-Crystalline Films on Epitaxial Graphene. Nat. Commun. 2014, 5, 4836.

17. Huang, R. Graphene: Show of Adhesive Strength. Nat. Nanotechnol. 2011, 6, 537-538.

18. Chung, K.; Lee, C. H.; Yi, G. C. Transferable GaN Layers Grown on ZnO Coated Graphene Layers for Optoelectronic Devices. Science 2010, 330, 655-657.

19. Chung, K.; Park, S. I.; Baek, H.; Chung, J. S.; Yi, G. C. High-Quality GaN Films Grown on Chemical Vapor-Deposited Graphene Films. NPG Asia Mater. 2012, 4, e24. 
20. Liu, H. J.; Wang, C. K.; Su, D.; Amrillah, T.; Hsieh, Y. H.; Wu, K. H.; Chen, Y. C.; Juang, J. Y.; Eng, L. M.; Jen, S. U.; Chu, Y. H. Flexible Heteroepitaxy of $\mathrm{CoFe}_{2} \mathrm{O}_{4} /$ Muscovite Bimorph with Large Magnetostriction. ACS Appl. Mat. Interface 2017, 8, 7297-7304.

21. Li, C. I.; Lin, J. C.; Liu, H. J.; Chu, M. W.; Chen, H. W.; Ma, C. H.; Tsai, C. Y.; Huang, H. W.; Lin, H. J.; Liu, H. L.; Chiu, P. W.; Chu, Y. H. Van Der Waal Epitaxy of Flexible and Transparent $\mathrm{VO}_{2}$ Flm on Muscovite. Chem. Mater. 2016, 28, 3914-3919.

22. Ma, C. H.; Lin, J. C.; Liu, H. J.; Do, T. H.; Zhu, Y. M.; Ha, T. D.; Zhan, Q.; Juang, J. Y.; He, Q.; Arenholz, E.; Chiu, P. W.; Chu, Y. H. Van Der Waals Epitaxy of Functional $\mathrm{MoO}_{2}$ Film on Mica for Flexible Electronics. Appl. Phys. Lett. 2016, 108, 253104.

23. Wu, P. C.; Chen, P. F.; Do, T. H.; Hsieh, Y. H.; Ma, C. H.; Ha, T. D.; Wu, K. H.; Wang, Y. J.; Li, H. B.; Chen, Y. C.; Juang, J. Y.; Yu, P.; Eng, L. M.; Chang, C. F.; Chiu, P. W.; Tjeng, L. H.; Chu, Y. H. Heteroepitaxy of $\mathrm{Fe}_{3} \mathrm{O}_{4} /$ Muscovite: A New Perspective for Flexible Spintronics. ACS Appl. Mater. Interfaces 2016, 8, 3379433801.

24. Bitla, Y.; Chen, C.; Lee, H. C.; Do, T. H.; Ma, C. H.; Qui, L. V.; Huang, C. W.; Wu, W. W.; Chang, L.; Chiu, P. W.; Chu, Y. H. Oxide Heteroepitaxy for Flexible Optoelectronics. ACS Appl. Mater. Interfaces 2016, 8, 32401-32407.

25. Bozorth, R. M.; Tilden, E. F.; Williams, A. J. Anisotropy and Magnetostriction of Some Ferrites. Phys. Rev. 1955, 99, 1788-1798. 
26. Ederer, C.; Spaldin, N. A. Effect of Epitaxial Strain on the Spontaneous Polarization of Thin Film Ferroelectrics. Phys. Rev. Lett. 2005, 95, 257601.

27. Jang, H. W.; Baek, S. H.; Ortiz, D.; Folkman, C. M.; Das, R. R.; Chu, Y. H.; Shafer, P.; Zhang, J. X.; Choudhury, S.; Vaithyanathan, V.; Chen, Y. B.; Felker, D. A.; Biegalski, M. D.; Rzchowski, M. S.; Pan, X. Q.; Schlom, D. G.; Chen, L. Q.; Ramesh, R.; Eom, C. B. Strain-Induced Polarization Rotation in Epitaxial (001) $\mathrm{BiFeO}_{3}$ Thin Films. Phys. Rev. Lett. 2008, 101, 107602.

28. Schmitz-Antoniak, C.; Schmitz, D.; Borisov, P.; de Groot, F. M. F.; Stienen, S.; Warland, A.; Krumme, B.; Feyerherm, R.; Dudzik, E.; Kleemann, W.; Wende, H. Electric In-Plane Polarization in Multiferroic $\mathrm{CoFe}_{2} \mathrm{O}_{4} / \mathrm{BaTiO}_{3}$ Nanocomposite Tuned by Magnetic Fields. Nat. Commun. 2013, 4, 2051.

29. Aimon, N. M.; Liao, J.; Ross, C. A. Simulation of Inhomogeneous Magnetoelastic Anisotropy in Ferroelectric/Ferromagnetic Nanocomposites. Appl. Phys. Lett. 2012, $101,232901$.

30. Zhang, J. X.; Li, Y. L.; Schlom, D. G.; Chen, L. Q.; Zavaliche, F.; Ramesh, R.; Jia, Q. X. Phase-Field Model For Epitaxial Ferroelectric and Magnetic Nanocomposite Thin Films. Appl. Phys. Lett. 2007, 90, 052909.

31. Chen, L. Q. Phase-Field Method of Phase Transitions/Domain Structures in Ferroelectric Thin Films: A Review. J. Am. Ceram. Soc. 2008, 91, 1835-1844.

32. Chen, L. Q.; Shen, J. Applications of Semi-Implicit Fourier-Spectral Method to Phase Field Equations. Comput. Phys. Commun. 1998, 108, 147-158. 
33. Hu, S. Y.; Chen, L. Q. A Phase-Field Model for Evolving Microstructures with Strong Elastic Inhomogeneity. Acta Mater. 2001, 49, 1879-1890.

34. Khachaturyan, A. G. Theory of Structural Transformation in Solids; Wiley: New York, U.S., 1983.

35. Hu, J. M.; Nan, C. W. Electric-Field-Induced Magnetic Easy-Axis Reorientation in Ferromagnetic/Ferroelectric Layered Heterostructures. Phys. Rev. B 2009, 80, 1-11.

36. Zhang, J.; Fu, H.; Lu, W.; Dai, J.; Chan, H. L. W. Nanoscale Free-Standing Magnetoelectric Heteropillars. Nanoscale 2013, 5, 6747-6753.

37. Gao, X. S.; Bao, D. H.; Birajdar, B.; Habisreuther, T.; Mattheis, R.; Schubert, M. A.; Alexe, M.; Hesse, D. Switching of Magnetic Anisotropy in Epitaxial $\mathrm{CoFe}_{2} \mathrm{O}_{4}$ Thin Films Induced by $\mathrm{SrRuO}_{3}$ Buffer Layer. J. Phys. D: Appl. Phys. 2009, 42, 17006.

38. Jen, S. U.; Liu, C. C.; Chen, S. T. Magnetostriction Measurement of a Ferromagnetic Thin Film Using Digital Holographic Microscope. IEEE trans. on Magn. 2014, 50, 11.

39. du Tremolet de Lacheisserie, E.; Peuzin, J. C. Magnetostriction and Internal Stresses in Thin Films: The Cantilever Method Revisited. J. Magn. Magn. Mater. 1994, 136, 189 196.

40. Amrillah, T.; Vandrangi, S. K.; Bitla, Y.; Do, T. H.; Liao, S. C.; Tsai, C. Y.; Chin, Y. Y.; Liu, Y. T.; Lin, M. L.; He, Q.; Lin, H. J.; Lee, H. Y.; Lai, C. H.; Arenholz, E.; Juang, J. Y.; Chu, Y. H. Tuning the Magnetic Properties of Self-assembled $\mathrm{BiFeO}_{3}-$ $\mathrm{CoFe}_{2} \mathrm{O}_{4}$ Heteroepitaxy by Magnetostructural Coupling. Nanoscale 2016, 8, 88478854. 
41. Ma, Y. G.; Cheng, W. N.; Ning, M.; Ong, C. K. Magnetoelectric Effect in Epitaxial $\mathrm{Pb}\left(\mathrm{Zr}_{0.52} \mathrm{Ti}_{0.48}\right) \mathrm{O}_{3} / \mathrm{La}_{0.7} \mathrm{Sr}_{0.3} \mathrm{MnO}_{3}$ Composite Thin Film. Appl. Phys. Lett. 2007, 90, 152911.

42. Zhang, Y.; Deng, C.; Ma, J.; Lin, Y.; Nan, C. W. Enhancement in Magnetoelectric Response in $\mathrm{CoFe}_{2} \mathrm{O}_{4}-\mathrm{BaTiO}_{3}$ Heterostructure. Appl. Phys. Lett. 2008, 92, 062911.

43. Ryu, S.; Park, J. H.; Jang, H. M. Magnetoelectric coupling of [001]Oriented $\mathrm{Pb}\left(\mathrm{Zr}_{0.4} \mathrm{Ti}_{0.6}\right) \mathrm{O}_{3}-\mathrm{Ni}_{0.8} \mathrm{Zn}_{0.2} \mathrm{Fe}_{2} \mathrm{O}_{4}$ Multilayered Thin Films. Appl. Phys. Lett. 2007, 91, 142910.

44. Ryu, H.; Murugavel, P.; Lee, J. H.; Chae, S. C.; Noh, T. W.; Oh, Y. S.; Kim, H. J.; Kim, K. H.; Jang, J. H.; Kim, M.; Bae, C.; Park, J. G. Magnetoelectric Effects of Nanoparticulate $\mathrm{Pb}\left(\mathrm{Zr}_{0.52} \mathrm{Ti}_{0.48}\right) \mathrm{O}_{3}-\mathrm{NiFe}_{2} \mathrm{O}_{4}$ Composite Films. Appl. Phys. Lett. 2006, 89, 102907.

45. Yan, L.; Zhuo, M.; Wang, Z.; Yao, J.; Haberkorn, N.; Zhang, S.; Civale, L.; Li, J.; Viehland, D.; Jia, Q. X. Magnetoelectric Properties of Flexible $\mathrm{BiFeO}_{3} / \mathrm{Ni}$ Tapes. Appl. Phys. Lett. 2012, 101, 012908.

46. Wang, Z.; Yan, L.; Yang, Y.; Li, J. F.; Das, J.; Geiler, A. L.; Yang, A.; Chen, Y.; Harris, V. G.; Viehland, D. Magnetoelectric Effect in Crystallographically Textured $\mathrm{BaTiO}_{3}$ Films Deposited on Ferromagnetic Metallic Glass Foils. J. Appl. Phys. 2011, 109, 034102. 
47. Triet, N. M.; Trung, T. Q.; Hien, N. T. D.; Siddiqui, S.; Kim, D.; Lee, J. C.; Lee, N. E. A Flexible Magnetoelectric Field-Effect Transistor with Magnetically Responsive Nanohybrid Gate Dielectric Layer. Nano Res. 2015, 10, 3421-3429.

48. Chaix-Pluchery, O.; Cochard, C.; Jadhav, P.; Kreisel, J.; Dix, N.; Sanchez, F.; Fontcuberta, J. Strain Analysis of Multiferroic $\mathrm{BiFeO}_{3}-\mathrm{CoFe}_{2} \mathrm{O}_{4}$ Nanostructures by Raman Scattering. Appl. Phys. Lett. 2011, 99, 072901.

49. Liao, Y. Y.; Li, Y. W.; Hu, Z. G.; Chu, J. H. Temperature Dependent Phonon Raman Scattering of Highly $a$-axis Oriented $\mathrm{CoFe}_{2} \mathrm{O}_{4}$ Inverse Spinel Ferromagnetic Films Grown by Pulsed Laser Deposition. Appl. Phys. Lett. 2012, 100, 071905. 